\title{
Simulation of nucleation in almost hard-sphere colloids: The discrepancy between experiment and simulation persists
}

\author{
L. Filion, ${ }^{1, a, b)}$ R. Ni, ${ }^{1, b)}$ D. Frenkel, ${ }^{2}$ and M. Dijkstra ${ }^{1}$ \\ ${ }^{1}$ Soft Condensed Matter, Debye Institute for NanoMaterials Science, Utrecht University, Princetonplein 5 , \\ 3584 CC Utrecht, The Netherlands \\ ${ }^{2}$ Department of Chemistry, University of Cambridge, Cambridge CB2 1EW, United Kingdom
}

(Received 26 January 2011; accepted 9 March 2011; published online 4 April 2011)

\begin{abstract}
In this paper we examine the phase behavior of the Weeks-Chandler-Andersen (WCA) potential with $\beta \epsilon=40$. Crystal nucleation in this model system was recently studied by Kawasaki and Tanaka [Proc. Natl. Acad. Sci. U.S.A. 107, 14036 (2010)], who argued that the computed nucleation rates agree well with experiment, a finding that contradicted earlier simulation results. Here we report an extensive numerical study of crystallization in the WCA model, using three totally different techniques (Brownian dynamics, umbrella sampling, and forward flux sampling). We find that all simulations yield essentially the same nucleation rates. However, these rates differ significantly from the values reported by Kawasaki and Tanaka and hence we argue that the huge discrepancy in nucleation rates between simulation and experiment persists. When we map the WCA model onto a hard-sphere system, we find good agreement between the present simulation results and those that had been obtained for hard spheres [L. Filion, M. Hermes, R. Ni, and M. Dijkstra, J. Chem. Phys. 133, 244115 (2010); S. Auer and D. Frenkel, Nature 409, 1020 (2001)]. (C) 2011 American Institute of Physics. [doi:10.1063/1.3572059]
\end{abstract}

\section{INTRODUCTION}

In a recent paper, Kawasaki and Tanaka ${ }^{1}$ examined the crystal nucleation in an almost hard-sphere system using Brownian dynamics (BD) simulations. The nucleation rates reported in Ref. 1 appear to be in good agreement with those that were found in earlier light scattering experiments. ${ }^{2-4}$ This is in sharp contrast with previous simulation studies of hard spheres, which show a large discrepancy between the experimental and simulated rates for low volume fractions. ${ }^{5,6}$ In the present paper we revisit the system examined by Kawasaki and Tanaka in order to determine the origin of the difference between the simulated rates and, in particular, to clarify if there is indeed a discrepancy between the experimental and simulated nucleation rates. We study the system using a variety of simulation techniques, including brute force Brownian dynamics, umbrella sampling (US), and forward flux sampling (FFS).

Colloidal solutions consist of small particles suspended in another medium and are typically characterized by the dynamics of these suspended particles, i.e., colloidal particles exhibit Brownian motion. As a result, BD simulations are the natural choice to use when examining dynamical properties of colloidal systems, such as crystal nucleation. Brownian dynamics are based on a simplified version of Langevin dynamics and correspond to the "overdamped" limit. Specifically, in BD it is assumed that the particles' inertial motion is completely damped out by frictional forces. As a result, the motion of the particles is determined by the instantaneous forces acting on the colloid plus a stochastic, diffusive displace-

\footnotetext{
a) Author to whom correspondence should be addressed. Electronic mail: L.C.Filion@uu.nl.

b) These authors contributed equally to this work.
}

ment. However, unlike molecular dynamics (MD) simulations where an event driven formalism exists which allows one to apply MD to systems with hard-core interactions (see, e.g., Ref. 7), no such formalism exists for BD of hard particles. Hence, when Brownian dynamics are applied to hard-core interactions, the hard core is typically approximated. One such approximation is the Weeks-Chandler-Andersen (WCA) potential.

The WCA potential was introduced in 1971 in order to address the short-range repulsive part of the Lennard-Jones liquid separately from the longer range attractive tail. In contrast to the Lennard-Jones system, the phase diagram for the WCA potential consists simply of liquid and solid phases; i.e., the liquid-gas phase coexistence is not present in this model. The WCA potential ${ }^{8}$ is given by

$$
\beta U_{\mathrm{WCA}}(r)=\left\{\begin{array}{l}
4 \beta \epsilon\left(\left(\frac{\sigma}{r}\right)^{12}-\left(\frac{\sigma}{r}\right)^{6}+\frac{1}{4}\right) r / \sigma \leq 2^{1 / 6} \\
0 \\
r / \sigma>2^{1 / 6}
\end{array}\right.
$$

where $\sigma$ is a length scale, $\epsilon$ is the energy scale, and $\beta$ $=1 / k_{B} T$ where $k_{B}$ is Boltzmann's constant and $T$ is the temperature. Note that the WCA potential is simply the LennardJones potential where the cutoff is chosen such that only the repulsive part remains and the potential is shifted upward so that the minimum occurs at zero. A plot of this potential is shown in Fig. 1. The "hardness" of the interaction can be set by tuning the interaction strength, $\beta \epsilon$. In Ref. 1, Kawasaki and Tanaka studied a WCA model at an interaction strength $\beta \epsilon=40$, which corresponds to a low temperature.

This paper is organized as follows: in Sec. II we use freeenergy calculations to determine the phase diagram for this model, in Sec. III we describe the nucleation rates, in Sec. IV 


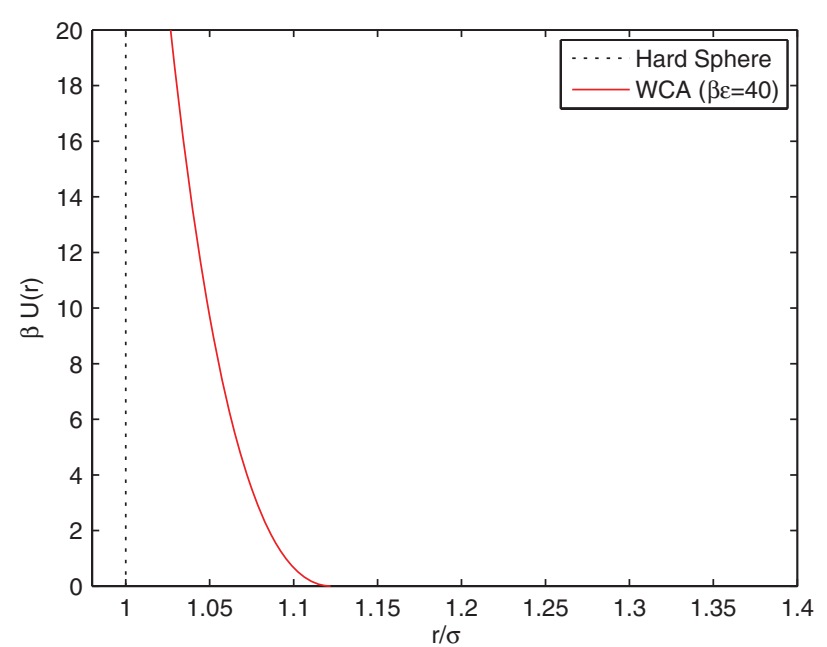

FIG. 1. The WCA potential and hard-sphere potential $\beta U(r)$ as a function of center-to-center distance $r$.

we compare our results to the previous work of Kawasaki and Tanaka ${ }^{1}$ and to hard-sphere crystal nucleation rates found both in simulations as well as light scattering experiments. Our conclusions are found in Sec. V.

\section{PHASE DIAGRAM}

To calculate the coexistence densities for the WCA potential, we used full free-energy calculations in combination with common tangent constructions. For the crystal phase, the excess free energy $F_{\text {ex }}$ was calculated using Einstein integration $^{10-12}$ at a density of $\rho \sigma^{3}=0.8$ for systems of $N=500,864,1372$, and 2048 particles. Note that the excess free energy is defined by $F_{\text {ex }}=F_{\text {tot }}-F_{\text {id }}$ where $F_{\text {tot }}$ is the total free energy and $F_{\text {id }}$ is the ideal gas free energy. Following Ref. 11, we plotted $\beta F_{\text {ex }} / N+\log N / N$ as a function of $1 / N$ and extrapolated to an infinite system yielding a free energy of $\beta F_{\text {tot }} / N=4.8975$. The free energy at other densities was determined using thermodynamic integration of the equation of state. ${ }^{12}$ The equation of state was determined using Monte Carlo NpT simulations with $N=4000$ particles. We note that no significant difference was found in the coexistence densities for equations of state determined using $N=1372$ and $N=4000$. To test our Einstein integration and integration over the equation of state, we determined the free energy at $\rho \sigma^{3}=0.9$ for $N=1372$ and integrated over the equation of state calculated for $N=1372$. The free energies agreed within $0.00046 k_{B} T$ per particle. The fluid chemical potential was determined using the Widom insertion technique $^{12}$ at $\rho \sigma^{3}=0.4$ with $N=4000$ and was found to be $\beta \mu=3.3173$; for $N=1372$ we find $\beta \mu=3.3194$. Again integration over the equation of state was used to determine the free energy as a function of density. To test the Widom insertion calculations, and our integration over the equation of state, we also calculated the chemical potential at $\rho \sigma^{3}=0.3$ for $N=1372$. The difference in the free energy at $\rho \sigma^{3}=0.3$ associated with the Widom insertions and integration over the equation of state results in a free-energy difference of $0.00075 k_{B} T$ per particle, and hence we concluded that the Widom insertions and integration over the equation of state

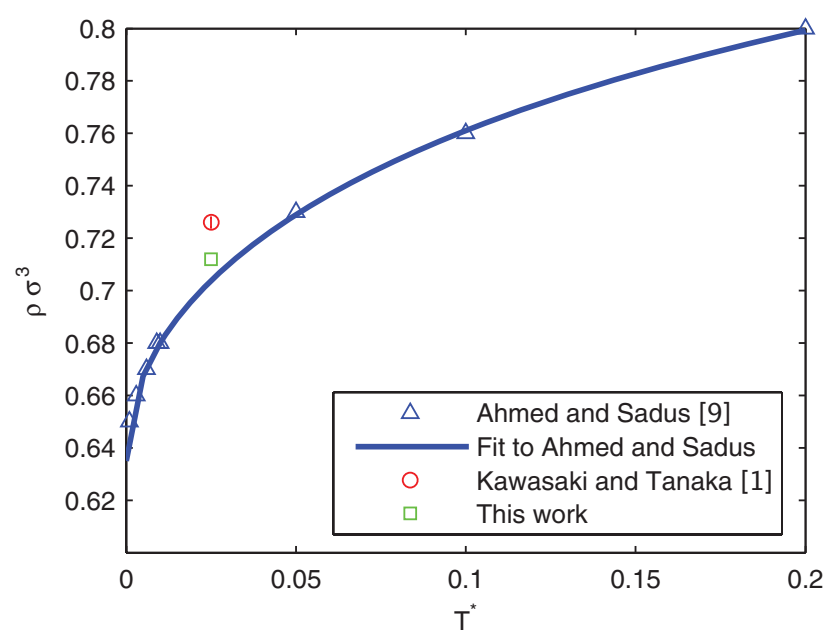

FIG. 2. The triangles correspond to the freezing number density $\left(\rho_{F}^{A}\right)$ from Ahmed and Sadus (Ref. 9) as a function of $T^{*}$ where $T^{*}=k_{B} T / \epsilon$. The fit corresponds to $\sigma \rho_{F}=0.635+0.473\left(T^{*}\right)^{1 / 2}-0.236 T^{*}$. The square corresponds to the freezing number density $\left(\rho_{F}^{*}\right)$ determined using full free-energy calculations as described in this paper. The circle corresponds to the freezing number density determined by Kawasaki and Tanaka (Ref. 1).

were correct. Using these free energies and common tangent constructions we find freezing and melting coexistence densities $\rho_{F}^{*} \sigma^{3}=0.712$ and $\rho_{M}^{*} \sigma^{3}=0.785$, respectively.

The phase diagram for the WCA potential has been examined previously by Ahmed and Sadus ${ }^{9}$ for a range of $T^{*}=1 / \beta \epsilon$ using a phenomenological method based on nonequilibrium MD simulations. The results of Ref. 9 for the freezing density are plotted in Fig. 2 . We find that their results for the freezing number density $\rho_{F}$ as a function of $\beta \epsilon$ fit well to $\rho_{F} \sigma^{3}=0.635+0.473\left(T^{*}\right)^{1 / 2}-0.236 T^{*}$. From this fit we approximate a freezing number density of $\rho_{F}^{A} \sigma^{3}=0.704$ at $\beta \epsilon=40$. We note that this is in good agreement with our predictions. Hence, our free-energy calculations support the phenomenological procedure of Ref. 9. However, we find that the nonequilibrium MD estimate of the freezing density is slightly lower than the true equilibrium coexistence density reported here. Additionally, Kawasaki and Tanaka ${ }^{1}$ found the freezing number density for $\beta \epsilon=40$ to be $\rho_{F}^{K} \sigma^{3}=0.725$. To locate the freezing point, these authors performed BD simulations of a face-centered-cubic (FCC) crystal and identified the density at which the crystal becomes mechanically unstable as the freezing density. ${ }^{13}$ Such calculations cannot be used to accurately determine the coexistence densities, but rather give an approximate lower bound for the melting density. As can be seen in Fig. 2, the freezing density estimated in Ref. 1 is some $2 \%$ higher than the value that we find using free-energy calculations.

\section{NUCLEATION RATES}

In this section we apply BD, US, and FFS to study the crystal nucleation of the WCA model. The methods for predicting nucleation rates have been discussed in detail in Ref. 5 and so only a short overview will be presented here. An overview of the state points discussed in this paper is found in Table I where we list for various pressures $\beta p \sigma^{3}$ 
TABLE I. Reduced pressure $\left(\beta p \sigma^{3}\right)$, reduced chemical potential difference between the fluid and solid phases $(\beta|\Delta \mu|)$, reduced number density of the metastable liquid $\rho_{\text {liq }} \sigma^{3}$, reduced number density of the solid phase $\rho_{\text {sol }} \sigma^{3}$, and the effective hard-sphere packing fraction $\phi^{\text {eff }}$ for the state points studied in this paper.

\begin{tabular}{lllll}
\hline \hline$\beta p \sigma^{3}$ & $\beta|\Delta \mu|$ & $\rho_{\text {liq }} \sigma^{3}$ & $\rho_{\text {sol }} \sigma^{3}$ & $\phi_{\text {eff }}$ \\
\hline 12.0 & 0.41 & 0.762 & 0.844 & 0.526 \\
13.0 & 0.54 & 0.775 & 0.858 & 0.535 \\
13.3 & 0.58 & 0.778 & 0.862 & 0.538 \\
13.4 & 0.59 & 0.780 & 0.863 & 0.539 \\
13.6 & 0.61 & 0.782 & 0.865 & 0.540 \\
13.9 & 0.65 & 0.785 & 0.868 & 0.542 \\
14.0 & 0.66 & 0.787 & 0.870 & 0.544 \\
14.4 & 0.71 & 0.791 & 0.874 & 0.547 \\
14.6 & 0.73 & 0.793 & 0.876 & 0.548 \\
\hline \hline
\end{tabular}

the corresponding chemical potential difference between the fluid and solid phases $|\beta \Delta \mu|$, the reduced number density of the metastable liquid phase $\rho_{\mathrm{liq}} \sigma^{3}$ and the stable solid phase $\rho_{\text {sol }} \sigma^{3}$, and the effective packing fraction $\phi_{\text {eff }}$ (as defined below).

In all of the simulations methods examined in this paper, an order parameter is needed to differentiate between liquidlike and solid-like particles and a cluster algorithm is used to identify the solid clusters. The order parameter we use is the local bond order parameter introduced by Ten Wolde et al. ${ }^{14,15}$ To measure the local bond order parameter a list of "neighbors" is determined for each particle where the neighbors of particle $i$ consist of all particles found within a radial distance $r_{c}$ of particle $i$. The total number of neighbors is denoted $N_{b}(i)$. For each particle $i$, a bond orientational order parameter $q_{l, m}(i)$ is then defined as

$$
q_{l, m}(i)=\frac{1}{N_{b}(i)} \sum_{j=1}^{N_{b}(i)} Y_{l, m}\left(\theta_{i, j}, \phi_{i, j}\right),
$$

where $Y_{l, m}(\theta, \phi)$ are the spherical harmonics, $m \in[-l, l]$ and $\theta_{i, j}$ and $\phi_{i, j}$ are the polar and azimuthal angles of the centerof-mass distance vector $\mathbf{r}_{i j}=\mathbf{r}_{j}-\mathbf{r}_{i}$ and $\mathbf{r}_{i}$ the position vector of particle $i$. Solid-like particles are identified as particles for which the number of connections per particle $\xi(i)$ is at least $\xi_{c}$ and where

$$
\xi(i)=\sum_{j=1}^{N_{b}(i)} H\left(d_{l}(i, j)-d_{c}\right),
$$

$H$ is the Heaviside step function, $d_{c}$ is the dot-product cutoff, and

$$
d_{l}(i, j)=\frac{\sum_{m=-l}^{l} q_{l, m}(i) q_{l, m}^{*}(j)}{\left(\sum_{m=-l}^{l}\left|q_{l, m}(i)\right|^{2}\right)^{1 / 2}\left(\sum_{m=-l}^{l}\left|q_{l, m}(j)\right|^{2}\right)^{1 / 2}}
$$

All other particles are identified as liquid-like. A cluster consists of all solid-like particles with at least one solid-like neighbor in the same cluster and so each particle can be a member of only a single cluster. We note that this order
TABLE II. Nucleation rates, $k \sigma^{5} / D_{0}$, obtained from $(N V T)$ Brownian dynamics simulations for various densities $\rho \sigma^{3}$ with $n_{t r}$ and $n_{e}$ the number of simulations and the number of observed nucleation events, respectively and $\langle t\rangle$ is the average waiting time for a nucleation event.

\begin{tabular}{ccccc}
\hline \hline$\rho \sigma^{3}$ & $n_{t r}$ & $n_{e}$ & $\langle t\rangle / \tau_{B}$ & $k \sigma^{5} / D_{0}$ \\
\hline 0.79228 & 5 & 5 & 13.8 & $1.4 \times 10^{-5}$ \\
0.78507 & 5 & 5 & 159 & $1.2 \times 10^{-6}$ \\
0.78153 & 10 & 10 & 260 & $7.3 \times 10^{-7}$ \\
0.77700 & 20 & 10 & 3282 & $5.8 \times 10^{-8}$ \\
0.77468 & 50 & 5 & 23340 & $8.1 \times 10^{-9}$ \\
\hline \hline
\end{tabular}

parameter depends on four parameters, namely, the nearest neighbor cutoff defined as $r_{c}$, the dot-product cutoff defined as $d_{c}$, the minimum number of solid-like neighbors required for a particle to be identified as crystalline denoted $\xi_{c}$, and the symmetry index for the bond orientational order parameter $l$. Since the nucleus is expected to have random hexagonal order we set $l=6$. Additionally, we used $d_{c}=0.7, \xi_{c}=6$ or 8 as identified and $r_{c}$ is always either $1.5 \sigma$ or $1.6 \sigma$ and is explicitly indicated in each section.

\section{A. Brownian dynamics}

Brownian dynamics is a simplified Langevin dynamics which can be used to describe the motion of Brownian particles. In Brownian dynamics simulations, the motion of each particle $i$ is described by ${ }^{16}$

$$
\frac{\mathrm{d} \mathbf{r}_{i}}{\mathrm{~d} t}=\frac{1}{m \gamma}\left[-\nabla_{i} U+\mathbf{W}_{i}(t)\right]
$$

where $\gamma$ and $\mathbf{W}_{i}(t)$ are the friction coefficient and the stochastic force of the solvent, $m$ is the mass of the particles, and $U$ is the potential energy of the system. They are linked through the dissipation-fluctuation theorem $\left\langle\mathbf{W}_{i}(t)\right.$ - $\left.\mathbf{W}_{j}\left(t^{\prime}\right)\right\rangle=6 m \gamma k_{B} T \delta_{i j} \delta\left(t-t^{\prime}\right)$ where $\delta$ is the Kronecker delta function. In our simulations, $\gamma$ and $m$ are both set to 1 and we use the time step $\Delta t=10^{-5} \tau_{B}$ to integrate Eq. (5). Note that $\tau_{B}$ is the Brownian time which is defined as $\tau_{B}=\sigma^{2} / D_{0}$ where $D_{0}$ is the diffusion coefficient of the particle in the infinitely dilute system.

To calculate the nucleation rates from Brownian dynamics simulations, we perform multiple independent simulations of systems with $N=4096$ particles and with the volume $V$ chosen such that the density of interest is acquired. Each simulation stops when a nucleation event happens, and the nucleation rate is determined by

$$
k=\frac{1}{\langle t\rangle V},
$$

where $\langle t\rangle$ is the average waiting time for a single nucleation event. Thus $\langle t\rangle=\sum_{i} t_{i} / n_{e}$ where $t_{i}$ is the simulation time of the independent simulation $i$ and where $n_{e}$ is the number of nucleation events observed. The results from our BD simulations for varying densities are shown in Table II.

Additionally, for $\rho \sigma^{3}=0.77000$, we performed 50 independent Brownian dynamics simulations. After a total simulation time $\sum_{i} t_{i}=116700 \tau_{B}$ we have not observed a single nucleation event in a system of $N=4096$ particles. Since 
nucleation is a rare event, the probability distribution of a nucleation event happening at time $t$ is an exponential distribution given by

$$
p(t)=\frac{1}{\langle t\rangle} \exp \left(-\frac{t}{\langle t\rangle}\right),
$$

where $\langle t\rangle$ is the average waiting time for a nucleation event. The probability of a nucleation event happening before time $t$ is $\int_{0}^{t} p(t) \mathrm{d} t=1-\exp (-t /\langle t\rangle)$. Thus for $\rho \sigma^{3}=0.77$, we can estimate the upper boundary for the nucleation rate. We find that if the nucleation rate is $4.85503 \times 10^{-9} D_{0} / \sigma^{5}$, the probability to observe a nucleation event before $116700 \tau_{B}$ in a system of $N=4096$ is $95 \%$. Additionally, if the nucleation rate is $1.48499 \times 10^{-9} D_{0} / \sigma^{5}$, this probability is $60 \%$.

\section{B. Umbrella sampling}

The crystal nucleation rate $k$ is related to the free-energy barrier $\Delta G(n)$ by $^{6}$

$$
k=A e^{-\beta \Delta G\left(n^{*}\right)},
$$

where

$$
A \approx \rho f_{n^{*}} \sqrt{\frac{\left|\beta \Delta G^{\prime \prime}\left(n^{*}\right)\right|}{2 \pi}},
$$

$n^{*}$ is the number of particles in the critical nucleus, $\rho$ is the number density of the supersaturated fluid, $f_{n^{*}}$ is the rate particles are attached to the critical cluster, and $\Delta G^{\prime \prime}$ is the second derivative of the Gibbs free-energy barrier. Hence, to determine the nucleation rate, we need to determine the Gibbs free-energy barrier $\Delta G(n)$ and the attachment rate $f_{n^{*}}$. In this section, we use umbrella sampling to determine the Gibbs free-energy barriers, and subsequently we calculate the crystal nucleation rates from these barriers.

The Gibbs free-energy barrier can be determined by ${ }^{17}$

$$
\beta \Delta G(n)=\text { constant }-\ln (P(n)),
$$

where $P(n)$ is the probability of observing a cluster of size $n$. We measure $P(n)$ using umbrella sampling. In US a biasing potential is added to the true interaction potential in order to facilitate the sampling of regions of configurational space which are inaccessible by traditional schemes, e.g., Metropolis Monte Carlo simulations. The typical US biasing potential for studying nucleation is given by ${ }^{14,18}$

$$
\beta U_{\text {bias }}\left(n\left(\mathbf{r}^{N}\right)\right)=\frac{\lambda}{2}\left(n\left(\mathbf{r}^{N}\right)-n_{C}\right)^{2},
$$

where $\lambda$ is a coupling parameter, $n\left(\mathbf{r}^{N}\right)$ is the size of the largest cluster associated with configuration $\mathbf{r}^{N}$, and $n_{C}$ is the targeted cluster size. It follows that the expectation value of an observable $A$ is given by

$$
\langle A\rangle=\frac{\left\langle 1 / W\left(n\left(\mathbf{r}^{N}\right)\right)\right\rangle_{\text {bias }}}{\left\langle 1 / W\left(n\left(\mathbf{r}^{N}\right)\right)\right\rangle_{\text {bias }}},
$$

where

$$
W(x)=e^{-\beta U_{\text {bias }}(x)} .
$$

This strategy is used to determine $P(n)$. A thorough description is given in Ref. 19.

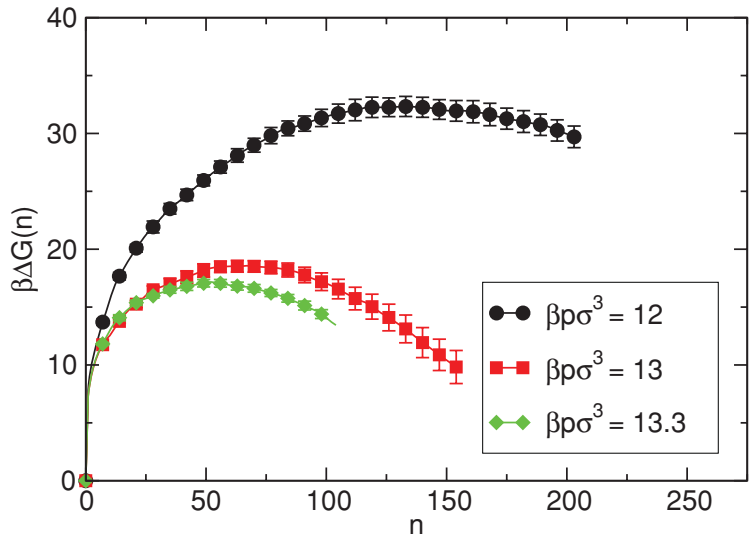

FIG. 3. Gibbs free-energy barriers $\beta \Delta G(n)$ as a function of cluster size $n$ as obtained from umbrella sampling MC simulations at reduced pressures $\beta p \sigma^{3}=12,13$, and 13.3 as labeled.

The attachment rate $f_{n^{*}}$ is related to the mean square deviation (MSD) of the cluster size $\left\langle\Delta n^{2}(t)\right\rangle=\left\langle\left(n(t)-n^{*}\right)^{2}\right\rangle$ at the top of the barrier by ${ }^{17}$

$$
f_{n^{*}}=\frac{1}{2} \frac{\left\langle\Delta n^{2}(t)\right\rangle}{t} .
$$

The MSD can be calculated by employing either a kinetic Monte Carlo (KMC) simulation or a MD simulation at the top of the barrier. For more information see Ref. 19.

For pressures $\beta p \sigma^{3}=12,13$, and 13.3, the free-energy barriers are shown in Fig. 3 and the attachment rates $f_{n^{*}}$ and nucleation rates $k$ are listed in Table III. Note that in these simulations we used a neighbor cutoff of $r_{c}=1.5 \sigma$ and coupling parameter $\lambda=0.2$.

\section{Forward flux sampling}

We use forward flux sampling to determine the nucleation rates for pressures $\beta p \sigma^{3}=12,13$, and 14 . The method is the same as the one described in Ref. 5 which was employed to determine the crystal nucleation rates for hard spheres and hence we will only give a brief overview here.

In FFS, phase space is mapped onto a reaction coordinate which is used to separate phase space by a sequence of interfaces $\left(\lambda_{0}, \lambda_{1}, \ldots \lambda_{N}\right)$ associated with increasing values $n\left(\mathbf{r}^{N}\right)$. In this case we use the order parameter described previously. The nucleation rate from the fluid phase $A$ to the solid phase

TABLE III. Nucleation rates, $k \sigma^{5} / D_{0}$, as obtained from $(N p T)$ umbrella sampling MC simulations at various pressures, $\beta p \sigma^{3}$, with $\rho \sigma^{3}$ the corresponding density of the supersaturated fluid. $\beta \Delta G\left(n^{*}\right)$ is the height of the free-energy barriers with $n^{*}$ the size of the critical cluster, and $\beta \Delta G^{\prime \prime}\left(n^{*}\right)$ and $f_{n^{*}} / D_{0}$ are the second order derivative and attachment rate at the top of the free-energy barrier, respectively.

\begin{tabular}{lcccccc}
\hline \hline$\beta p \sigma^{3}$ & $n^{*}$ & $f_{n^{*}} / D_{0}$ & $\beta \Delta G\left(n^{*}\right)$ & $\beta \Delta G^{\prime \prime}\left(n^{*}\right)$ & $\rho \sigma^{3}$ & $k \sigma^{5} / D_{0}$ \\
\hline 12 & 130 & 586.17 & 32.5 & 0.0015 & 0.762 & $5.23 \times 10^{-14}$ \\
13 & 60 & 319.05 & 18.5 & 0.0030 & 0.774 & $4.98 \times 10^{-8}$ \\
13.3 & 50 & 361.86 & 17.200 & 0.0030 & 0.777 & $2.08 \times 10^{-7}$ \\
\hline \hline
\end{tabular}


TABLE IV. Probabilities $P\left(\lambda_{i+1} \mid \lambda_{i}\right)$ for the interfaces used in calculating the nucleation rate for pressure $\beta p \sigma^{3}=12$.

\begin{tabular}{lcc}
\hline \hline $\mathrm{i}$ & $\lambda_{i}$ & $P\left(\lambda_{i} \mid \lambda_{i-1}\right)$ \\
\hline 2 & 20 & 0.133 \\
3 & 26 & 0.132 \\
4 & 34 & 0.107 \\
5 & 45 & 0.068 \\
6 & 60 & 0.066 \\
7 & 80 & 0.041 \\
8 & 110 & 0.036 \\
9 & 150 & 0.130 \\
10 & 200 & 0.317 \\
11 & 250 & 0.842 \\
\hline \hline
\end{tabular}

$B$ is then given by

$$
\begin{aligned}
& k_{A B}=\Phi_{A \lambda_{0}} P\left(\lambda_{N} \mid \lambda_{0}\right) \\
& =\Phi_{A \lambda_{0}} \prod_{i=0}^{N-1} P\left(\lambda_{i+1} \mid \lambda_{i}\right),
\end{aligned}
$$

where $\Phi_{A \lambda_{0}}$ is the steady-state flux of trajectories leaving the $A$ state and crossing the interface $\lambda_{0}$ in a volume $V$, and $P\left(\lambda_{i+1} \mid \lambda_{i}\right)$ is the probability that a configuration starting at interface $\lambda_{i}$ will reach interface $\lambda_{i+1}$ before it returns to the fluid $(A)$. As in Ref. 5 we regroup the elements of the rate calculation such that

$$
k_{A B}=\tilde{\Phi}_{A \lambda_{1}} \prod_{i=1}^{N-1} P\left(\lambda_{i+1} \mid \lambda_{i}\right),
$$

where

$$
\tilde{\Phi}_{A \lambda_{1}}=\Phi_{A \lambda_{0}} P\left(\lambda_{1} \mid \lambda_{0}\right) .
$$

If $\lambda_{1}$ is chosen such that it is a relatively rare event for trajectories starting in $A$ to reach $\lambda_{1}$, then

$$
\tilde{\Phi}_{A \lambda_{1}} \approx \frac{1}{\left\langle t_{A \lambda_{1}}\right\rangle V}
$$

where $\left\langle t_{A \lambda_{1}}\right\rangle$ is the average time it takes a trajectory in $A$ to reach $\lambda_{1}$. This approximation has been discussed in more detail in Ref. 5.

The dynamics in the forward flux sampling simulations were approximated using kinetic Monte Carlo simulations with a step size of $\Delta_{\mathrm{KMC}}=0.05 \sigma$ and measuring the order parameter every $\Delta t_{\text {ord }}=2 \mathrm{MC}$ cycles. The nearest neighbor cutoff for the order parameter was taken to be $r_{c}=1.5 \sigma$. The probabilities $P\left(\lambda_{i} \mid \lambda_{i-1}\right)$ of going from interface $\lambda_{i-1}$ to $\lambda_{i}$ required in the forward flux sampling rate calculation for pressures $\beta p \sigma^{3}=12,13$, and 14 are given in Tables IV,V, and VI, respectively. The resulting rates in terms of the short-time diffusion coefficient $D_{0}$ are given in Table VII .

\section{DISCUSSION}

In this section we compare our predicted nucleation rates to previous theoretical and experimental studies. In Fig. 4 we
TABLE V. Probabilities $P\left(\lambda_{i+1} \mid \lambda_{i}\right)$ for the interfaces used in calculating the nucleation rate for pressure $\beta p \sigma^{3}=13$.

\begin{tabular}{lcc}
\hline \hline $\mathrm{i}$ & $\lambda_{i}$ & $P\left(\lambda_{i} \mid \lambda_{i-1}\right)$ \\
\hline 2 & 20 & 0.132 \\
3 & 30 & 0.124 \\
4 & 40 & 0.193 \\
5 & 60 & 0.132 \\
6 & 100 & 0.166 \\
7 & 150 & 0.633 \\
\hline \hline
\end{tabular}

show our predicted WCA crystal nucleation rates and compare them with those found in Ref. 1. Note that the nucleation rates shown in Fig. 4 (and Fig. 6) cannot be obtained directly from Ref. 1 as there is a mistake in that paper regarding the mapping from effective packing fraction units to number densities. ${ }^{13,20}$ We first note that our BD results match well with previous $\mathrm{BD}$ nucleation rates. ${ }^{1}$ We also note that the uncertainty in the BD results is approximately 1 order of magnitude and the uncertainty in the US and FFS results is approximately 2 orders of magnitude. Within this uncertainty, the BD, US, and FFS nucleation rates all agree. This is consistent with a recent study ${ }^{5}$ on hard spheres which found that molecular dynamics and FFS rates agreed well with the US rates of Auer and Frenkel. ${ }^{6}$

We note that the US and FFS simulations were performed at constant pressure, i.e., in an $N p T$ ensemble, while the BD simulations were at constant volume $(N V T)$. While we have not examined in detail the nuclei appearing in these simulations, no significant difference was found between the nuclei forming in the BD simulations and the nuclei forming in the FFS and US simulations. This question was addressed in more detail in a recent nucleation study on hard spheres. ${ }^{5}$ In that case, the radius of gyration tensor of the resulting clusters was measured as a function of cluster size for constant volume molecular dynamics simulations, and constant pressure FFS, and US simulations. No difference between the resulting nuclei was found. Additionally, in an $N V T$ ensemble, the formation of a nucleus depletes the number of particles in the fluid and lowers slightly the number density of the fluid. However, when the system size is sufficiently large, this effect is negligible. While this effect was not studied in this paper, it was examined by Kawasaki and Tanaka ${ }^{1}$ who found that the nucleation rates for this model (i.e., the WCA potential) at high supersaturation converged for approximately 4000 particles. For lower supersaturations, we can approximate the effect of the system size by determining the number density of the fluid when a critical nucleus is present at fixed volume. For a system containing $N=4096$ particles, at the lowest

TABLE VI. Probabilities $P\left(\lambda_{i+1} \mid \lambda_{i}\right)$ for the interfaces used in calculating the nucleation rate for pressure $\beta p \sigma^{3}=14$.

\begin{tabular}{lll}
\hline \hline $\mathrm{i}$ & $\lambda_{i}$ & $P\left(\lambda_{i} \mid \lambda_{i-1}\right)$ \\
\hline 2 & 40 & 0.164 \\
3 & 70 & 0.453 \\
4 & 100 & 0.847 \\
\hline \hline
\end{tabular}


TABLE VII. Nucleation rate $k \sigma^{5} / D_{0}$, flux $\Phi_{A \lambda_{1}}$, and $P\left(\lambda_{B} \mid \lambda_{1}\right)$ at various pressures $\beta p \sigma^{3}$ as obtained by $(N p T)$ forward flux sampling.

\begin{tabular}{llll}
\hline \hline$\beta p \sigma^{3}$ & $\Phi_{A \lambda_{1}} \sigma^{5} / D_{0}$ & $P\left(\lambda_{B} \mid \lambda_{1}\right)$ & $k \sigma^{5} / D_{0}$ \\
\hline 12 & $2.96 \times 10^{-6}$ & $4.32 \times 10^{-10}$ & $1.27 \times 10^{-15}$ \\
13 & $1.10 \times 10^{-5}$ & $4.38 \times 10^{-5}$ & $4.80 \times 10^{-10}$ \\
14 & $1.06 \times 10^{-5}$ & $6.29 \times 10^{-2}$ & $6.69 \times 10^{-7}$ \\
\hline \hline
\end{tabular}

density we studied using $\mathrm{BD}$ simulations, namely, $\rho \sigma^{3}$ $=0.775$, we find the fluid density to be approximately $\rho \sigma^{3}$ $=0.774$ when a critical nucleus containing 60 particles is present. As a result, we expect at the very most a horizontal error bar of 0.001 in the number density of the BD rates. Hence, we do not expect a significant effect from the system size in our BD simulations.

In Fig. 5 we compare our predicted WCA rates with the crystal nucleation rates of hard spheres as a function of supersaturation, i.e., the chemical potential difference between the bulk crystal and the supersaturated fluid $(\Delta \mu)$. We find good agreement between the nucleation rates in these two systems.

We further compare our WCA results with those of the hard-sphere system examined in Ref. 5 and the experimental light scattering results from Refs. 2-4. To do this, we scale our WCA results in terms of an effective packing fraction in the same manner as is done experimentally. Specifically, we scale the freezing number density of the WCA model $\left(\rho_{F} \sigma^{3}\right.$ $=0.712$ ) to the freezing packing fraction of hard spheres. Note that in literature there is a range of freezing packing fractions for hard spheres, namely, $0.491 \leq \phi_{F}^{H S} \leq 0.494$ (see, e.g., Refs. 12,21, and 22). Here we follow Frenkel and Smit ${ }^{12}$ which we believe to be the most accurate. In their work, finite size effects are taken into consideration when calculating the free energy of the FCC crystal, i.e., they use the result from Ref. 11. In addition, the Speedy equations of state for the solid

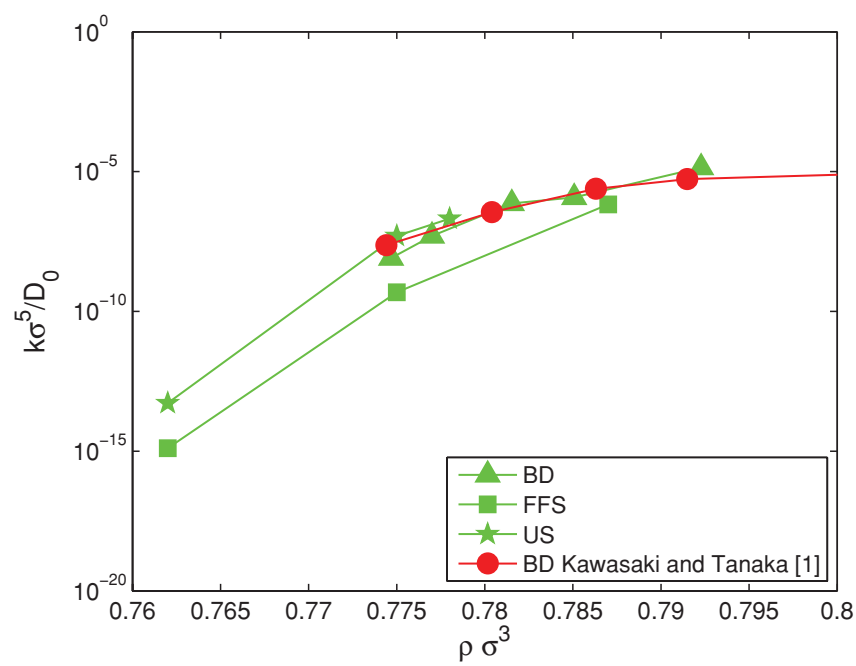

FIG. 4. Crystal nucleation rates $k \sigma^{5} / D_{0}$ as a function of number density $\rho \sigma^{3}$ where $D_{0}$ is the short-time diffusion coefficient. While we have not included error bars in this plot, note that the uncertainty in the US and FFS nucleation rates is approximately 2 orders of magnitude while the uncertainty in the BD results is approximately 1 order of magnitude. Note also that the US and FFS simulations were performed at constant pressure $(N p T)$ while the BD simulations were at constant volume $(N V T)$.

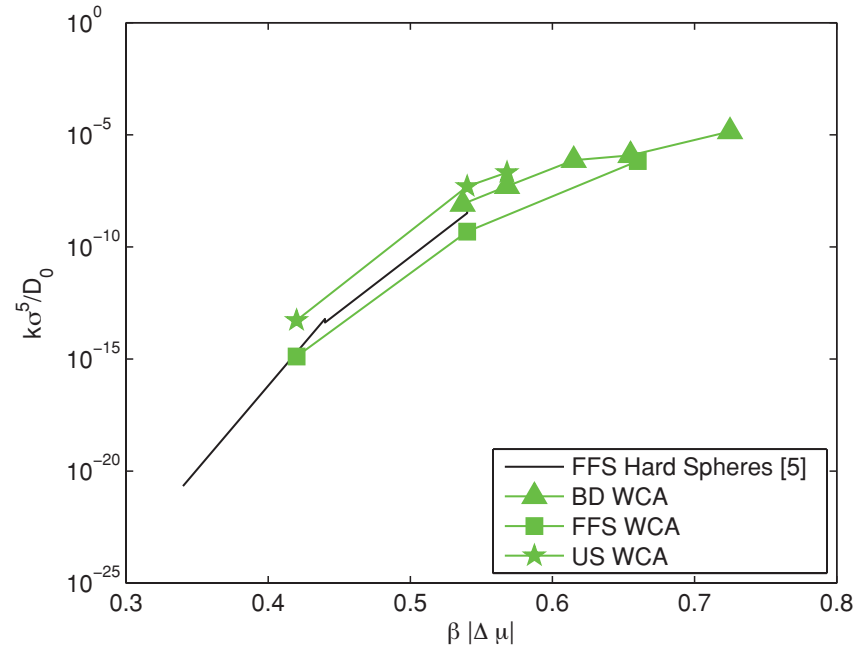

FIG. 5. Crystal nucleation rates $k \sigma^{5} / D_{0}$ as a function of supersaturation $\beta|\Delta \mu|$ where $D_{0}$ is the short-time diffusion coefficient. The hard-sphere (HS) nucleation rates are taken from Ref. 5.

and fluid phase were employed. ${ }^{23,24}$ The resulting freezing packing fraction is found to be $\phi_{F}^{H S}=0.492 .^{12}$ The WCA nucleation rates $k \sigma_{\text {eff }} / D_{0}$ scaled to $\phi_{F}^{H S}=0.492$ are compared to the hard-sphere results in Fig. 6 where $\sigma_{\text {eff }}$ is the size of a hard-sphere particle which has the same freezing number density as the WCA model. We stress here that any error in the freezing coexistence results in a horizontal shift in the nucleation rates. Hence, in addition to an uncertainty of approximately 2 orders of magnitude in the nucleation rates, there is an additional uncertainty of approximately $\Delta \phi_{\text {error }}= \pm 0.005$ in the effective packing fractions. Thus, within these error bars, we find good agreement between our predicted hardsphere and WCA crystal nucleation rates.

Previous studies ${ }^{25,26}$ have shown that softness in the potential increases the nucleation rate, however, this cannot be

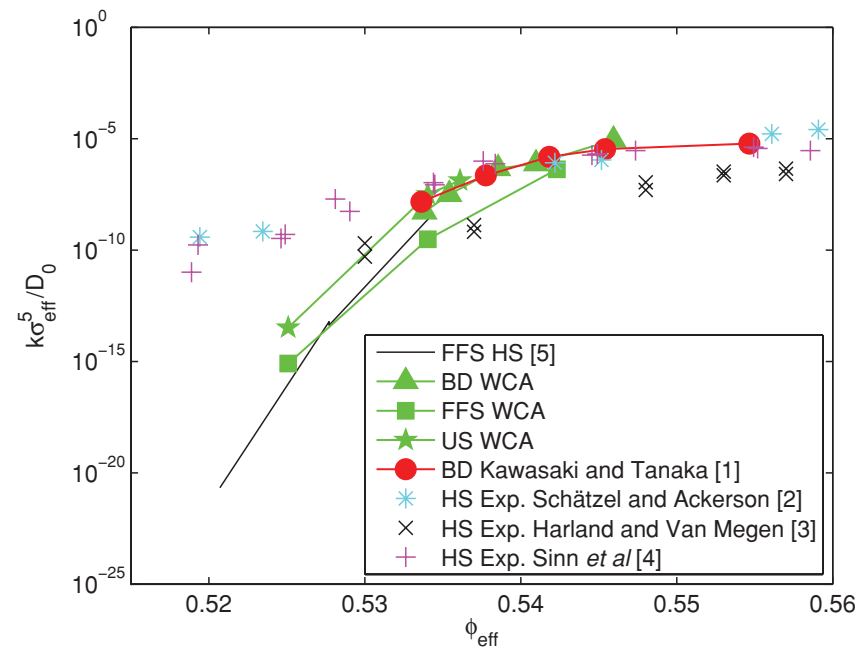

FIG. 6. Crystal nucleation rates $k \sigma_{\text {eff }}^{5} / D_{0}$ as a function of effective packing fraction $\phi_{\text {eff }}=\frac{\pi}{6} \rho \sigma_{\text {eff }}^{3}$ where $D_{0}$ is the short-time diffusion coefficient and $\rho$ is the number density of the fluid. Note that $\sigma_{\text {eff }}$ is the size of a hard-sphere particle which has the same freezing number density as the WCA model. The HS nucleation rates are taken from Ref. 5. 
confirmed from our predictions as the uncertainty in the nucleation rates is too large. We stress that the experimental hard-sphere nucleation rates differ significantly from our predicted rates for low supersaturations. This is in contrast to the results presented in Ref. 1 where good agreement was found between the WCA rates and the light scattering experimental nucleation rates. This difference originates from the freezing number density which was used to map the WCA number densities to effective packing fractions. As described in Sec. II, in this paper we have determined the freezing densities using full free-energy calculations which are known to be very accurate. In contrast, the method used in Ref. 1 appears to yield results that differ significantly from the "exact" coexistence densities.

The large difference between the nucleation rates when plotted in terms of effective packing fractions emphasizes one possible problem in the comparison between the experimental and simulated nucleation rates: the determination of the effective packing fractions. A difference of $1-2 \%$ in the freezing density has a significant effect on the position of the dropoff of the nucleation rates. Whereas it is straightforward to evaluate the correct effective volume fractions in simulations, the procedure required to deduce the same information from experiments is more subtle. Hence, part of the discrepancy between the computed and measured crystal-nucleation rates of "hard-sphere" colloids may be due to a small difference in the definition of the effective packing fraction. Yet, this is certainly not the whole story: the very large discrepancy between experimental and numerical nucleation rates at lower densities cannot be accounted for by a simple rescaling of the density axis. Hence, unlike Kawasaki and Tanaka, we conclude that the discrepancy between simulation and experiment is as large as ever, and still unexplained

\section{CONCLUSIONS}

In conclusion, we have examined the crystal nucleation of particles interacting with the WCA potential with $\beta \epsilon$ $=40$ using Brownian dynamics, umbrella sampling, and forward flux sampling. As in Ref. 5, we find good agreement between the nucleation rates predicted using these different methods. Additionally, we find that the nucleation rates predicted for the WCA model agree well with those of hard spheres as a function of the effective packing fraction $\phi_{\text {eff }}$ defined such that $\phi_{\text {eff }}$ at freezing matches that of hard spheres.

\section{ACKNOWLEDGMENTS}

We would like to thank H. Tanaka for many useful conversations. L.F., R.N., and M.D. acknowledge financial support from the NWO-VICI grant and the high potential programme from Utrecht University. D.F. acknowledges financial support from the Royal Society of London (Wolfson Merit Award) and from the ERC (Advanced Grant agreement 227758).

${ }^{1}$ T. Kawasaki and H. Tanaka, Proc. Natl. Acad. Sci. U.S.A. 107, 14036 (2010).

${ }^{2}$ K. Schätzel and B. J. Ackerson, Phys. Rev. E 48, 3766 (1993).

${ }^{3}$ J. L. Harland and W. van Megen, Phys. Rev. E 55, 3054 (1997).

${ }^{4}$ C. Sinn, A. Heymann, A. Stipp, and T. Palberg, Prog. Colloid Polym. Sci. 118, 266 (2001).

${ }^{5}$ L. Filion, M. Hermes, R. Ni, and M. Dijkstra, J. Chem. Phys. 133, 244115 (2010).

${ }^{6}$ S. Auer and D. Frenkel, Nature (London) 409, 1020 (2001).

${ }^{7}$ B. J. Alder and T. E. Wainwright, J. Chem. Phys. 31, 459 (1959).

${ }^{8}$ J. D. Weeks, D. Chandler, and H. C. Andersen, J. Chem. Phys. 54, 5237 (1971).

${ }^{9}$ A. Ahmed and R. J. Sadus, Phys. Rev. E 80, 061101 (2009).

${ }^{10}$ D. Frenkel and A. J. C. Ladd, J. Chem. Phys. 81, 3188 (1984).

${ }^{11}$ J. M. Polson, E. Trizac, S. Pronk, and D. Frenkel, J. Chem. Phys. 112, 5339 (2000).

${ }^{12}$ D. Frenkel and B. Smit, Understanding Molecular Simulation: From Algorithms to Applications (Academic, San Diego, CA, 2002).

${ }^{13}$ T. Kawasaki and H. Tanaka, private communication (2010-2011).

${ }^{14}$ P. ten Wolde, M. J. Ruiz-Montero, and D. Frenkel, Faraday Discuss. 104, 93 (1996).

${ }^{15}$ P. R. ten Wolde, Ph.D. thesis, University of Amsterdam, 1998.

${ }^{16}$ M. Allen and D. Tildesley, Computer Simulation of Liquids (Oxford University Press, New York, 1987).

${ }^{17}$ S. Auer and D. Frenkel, J. Chem. Phys. 120, 3015 (2004).

${ }^{18}$ P. R. ten Wolde, M. J. Ruiz-Montero, and D. Frenkel, J. Chem. Phys. 104, 9932 (1996).

${ }^{19}$ S. Auer, Ph.D. thesis, University of Amsterdam, 2002.

${ }^{20}$ The number densities appearing in Figs. 4 and 6 were obtained directly via communications with the authors of Ref. 1.

${ }^{21}$ R. L. Davidchack and B. B. Laird, J. Chem. Phys. 108, 9452 (1998).

${ }^{22}$ W. G. Hoover and F. H. Ree, J. Chem. Phys. 49, 3609 (1968).

${ }^{23}$ R. J. Speedy, J. Phys.: Condens. Matter 10, 4387 (1998).

${ }^{24}$ R. J. Speedy, J. Phys.: Condens. Matter 9, 8591 (1997).

${ }^{25}$ S. Auer and D. Frenkel, J. Phys.: Condens. Matter 14, 7667 (2002).

${ }^{26}$ S. Auer, W. C. K. Poon, and D. Frenkel, Phys. Rev. E 67, 020401 (2003). 